\title{
Kernos
}

Revue internationale et pluridisciplinaire de religion grecque antique

$30 \mid 2017$

Varia

\section{Détroits, isthmes, passages : paysages « sous le joug » de Poséidon}

\section{François de Polignac}

\section{(2) OpenEdition \\ Journals}

Édition électronique

URL : http://journals.openedition.org/kernos/2488

DOI : 10.4000/kernos.2488

ISSN : 2034-7871

Éditeur

Centre international d'étude de la religion grecque antique

Édition imprimée

Date de publication : 1 octobre 2017

Pagination : 67-83

ISSN : 0776-3824

\section{Référence électronique}

François de Polignac, « Détroits, isthmes, passages : paysages «sous le joug » de Poséidon », Kernos

[En ligne], 30 | 2017, mis en ligne le 01 octobre 2019, consulté le 18 septembre 2020. URL : http:// journals.openedition.org/kernos/2488; DOI : https://doi.org/10.4000/kernos.2488

Ce document a été généré automatiquement le 18 septembre 2020.

Kernos 


\title{
Détroits, isthmes, passages : paysages « sous le joug » de Poséidon
}

\author{
François de Polignac
}

1 Dans deux articles publiés dans la Revue archéologique, respectivement en 1944 et 1952, et consacré pour le premier aux « Géants de la mer » et pour le second aux « Génies des passes et des défilés ", Francis Vian avait attiré l'attention sur toute une série de personnages mythologiques caractérisés par leur taille gigantesque ou leur force exceptionnelle et associés à Poséidon" ${ }^{1}$. Parmi les "Géants ", l'historien avait identifié Géraistos dont le nom évoque une série de sanctuaires, de cultes, de fêtes, voire de noms de mois consacrés à un Poséidon Géraistios connu en premier lieu par son important sanctuaire à la pointe sud de l'Eubée, mais aussi à Trézène, à Cos et au Ténare ; Pélôr ou Pélôros, autre géant en interaction avec le Poséidon Pétraios ou Lytaios de Thessalie, l'ouverture de la vallée du Tempé pratiquée par le dieu et la célébration qui s'en suit des Pélôria; et un Aigaiôn, figure polymorphe parfois assimilée au « Cent Bras» Briarée, localisé en Eubée mais dont une tradition plaçait la tombe à l'embouchure du Rhyndacos, en Phrygie. Dans une optique fortement marquée par l'interprétation naturaliste, Vian voyait dans ces trois personnages «des êtres monstrueux qui personnifiaient les tempêtes de l'équinoxe et en même temps les forces néfastes de l'hiver $»^{2}$. En partie pour répondre aux critiques formulées par Lucien Lerat à propos de l'interprétation des Géraistai ${ }^{3}$, Francis Vian reprit l'étude de ces figures dans son second article en y adjoignant d'autres pour mettre l'accent sur leur qualité de gardiens de passages délicats : caps, défilés montagneux, isthmes. À Géraistos, pour les caps Géraistos et Ténare, Pélôr pour la vallée du Tempé, Aigaiôn, aux bouches du Rhyndacos, vinrent ainsi s'adjoindre des personnages aussi divers qu'Alkyoneus, géant vaincu par Héraclès soit à Cos, soit sur l'Isthme de Corinthe, et Alkyonê, nymphe qui engendra de Poséidon une des dynasties royales de Trézène; le géant Polybotès vaincu par Poséidon à Cos et recouvert par l'île de Nisiros; Skirôn et Kerkyon, deux des brigands parfois représentés comme fils du dieu et successivement vaincus par Thésée près de Mégare et à Éleusis ; et enfin les Gêgéneis, les « Nés de la terre » de Cyzique que 
les Argonautes exterminent chez Apollonios de Rhodes, et d'autres encore. Néanmoins, malgré l'association de tous ces personnages à Poséidon sous une forme ou une autre, la catégorie de ces " génies " perd en cohérence ce qu'elle gagne en extension : rattachée à des situations topographiques très dissemblables, ainsi qu'à de multiples divinités secondaires assez disparates («nymphes ", "Terre Mère» et similaire, "déesse de l'accouchement »), elle ne corrobore que faiblement l'hypothèse de Francis Vian selon laquelle il existait une corrélation forte entre « rites de passage et génies des passages » 4 .

2 Cependant, même si elle s'est traduite de manière trop vague dans le dernier article de Vian, l'idée d'une articulation entre un certain type de lieux, une certaine catégorie de personnages, souvent de taille gigantesque, et le dieu Poséidon mérite d'être approfondie afin de voir si, en s'attachant à définir un type particulier de "paysage religieux », on peut aussi identifier un trait fondamental de la présence et de l'action du dieu $^{5}$. Encore faut-il y mettre plusieurs conditions : la similitude ou l'analogie des lieux concernés doit être établie à partir des sources antiques elles-mêmes et non évaluées à partir des impressions de l'observateur moderne; de même, les figures monstrueuses qui y sont évoquées doivent partager des analogies fonctionnelles vérifiables et non pas une simple ressemblance superficielle. Mais la démarche paraît d'autant plus utile que, des dieux grecs, Poséidon est certainement celui qui a le moins fait l'objet d'analyses fondées sur la mise en relation des manières d'agir, des sphères d'intervention et des pratiques cultuelles, sans laquelle la place de la divinité dans le réseau des puissances qui organisent le monde et régissent la société humaine ne peut être mise en lumière. Dans la lignée des travaux fondateurs de Jean-Pierre Vernant et de Marcel Detienne, les études se sont multipliées, ces dernières décennies, qui ont montré qu'une divinité ne peut être comprise sur le mode de l'assignation de fonctions bien définies et de traits immuables, mais bien comme l'exercice d'une puissance dans des contextes dont les configurations dessinent un champ d'action multiforme. Apollon, Hermès, Aphrodite, Artémis, dernièrement Héra, ont ainsi été l'objet d'études dont le but déclaré n'est pas de faire l'inventaire de toutes les interventions des divinités concernées, ni de les figer dans une définition statique qui engloberait une fois pour toutes leurs différents aspects, mais bien plutôt de montrer comment chaque puissance divine se définit par sa façon particulière d'interagir et de se positionner par rapport aux autres puissances dans le domaine du règne de Zeus ${ }^{6}$.

Or, pour des raisons que l'on peut tenter d'élucider, Poséidon a été jusqu'à présent laissé en marge de ce courant d'étude. Il est vrai que le dieu a mauvaise réputation. Brutal et colérique, il déchaîne des forces terrifiantes ; il a, on le verra, de bien étranges fréquentations, en raison de ses accointances avec des êtres aussi peu recommandables que les Cent-Bras monstrueux, ou des géants de toute sorte souvent emplis d'hybris, ou encore des Cyclopes querelleurs. Il fait en outre figure de dieu plus ou moins déchu. Il existe en effet un courant d'interprétation qui tend à placer l'âge d'or de Poséidon à l'âge du Bronze, et plus précisément à l'époque mycénienne. Cette tendance est déjà perceptible dans l'ouvrage où Fritz Schachermeyr avait cherché à rattacher chacune des fonctions du dieu à une phase bien précise de l'histoire grecque : sa démonstration met clairement l'accent sur les hautes époques, en particulier l'époque mycénienne, par exemple pour toutes les fonctions liées à la maitrise du cheval et du char. Le Poséidon d'époque historique apparait dès lors comme une survivance de conceptions religieuses d'un âge antérieur'. L'analyse récente de Charles Doyen est encore plus 
tranchée : en se fondant sur l'étude des tablettes de Pylos, elle fait de Poséidon le « dieu souverain" de l'époque mycénienne, détrôné lorsque la souveraineté de Zeus fut instaurée à la faveur des changements sociaux, politiques et religieux consécutifs à la chute des palais ${ }^{8}$. Et nous verrons que même lorsque Marcel Detienne et Jean-Pierre Vernant s'intéressèrent à Poséidon dans le cadre de leurs recherches sur la mêtis des Grecs, dans une perspective évidemment totalement étrangère à l'interprétation généticienne des auteurs précédents, ils ne donnèrent qu'un rôle secondaire, voire passif, au dieu, qui fait figure de puissance relativement frustre par comparaison avec l'inventivité technique d'Athéna - on serait presque tenté de parler d'un Poséidon " primitif » par rapport à la « modernité » de la déesse ${ }^{9}$. Plus récemment, le travail très complet de Ioannis Mylonopoulos sur les cultes de Poséidon dans le Péloponnèse échappe à ce travers et restitue bien l'importance du dieu dans la péninsule. Il reste cependant dépendant d'une perspective plus analytique qu'organique des fonctions du dieu - dans les domaines des séismes, de la mer, du cheval, de la fertilité, de la politique, pour ne prendre que ces exemples - en les présentant, ainsi que les épiclèses, sous la traditionnelle forme juxtaposée ${ }^{10}$.

Également étrangère à la perspective généticienne, la présente étude entend contribuer à modifier cette vision du dieu et mettre en lumière certains des ressorts, modalités et contextes de ses interventions. Pour ce faire, elle vise à dessiner les contours d'un "paysage poséidonien » qui, par-delà la diversité des situations, permette d'identifier quelques traits fondamentaux des modes d'action du dieu.

\section{Ouvrir et fermer : naissance des détroits}

Plusieurs auteurs grecs et latins d'époque romaine mentionnent l'existence, sur la rive continentale du détroit de Messine, d'une «colonne des Rhégiens » ou «colonne de

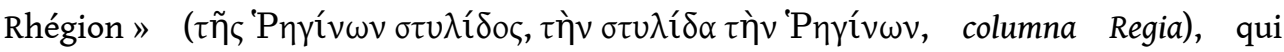
semble avoir été située à l'entrée nord du détroit, à l'endroit le plus resserré du passage entre le continent et la Sicile ${ }^{11}$. Les données fournies par ces auteurs permettent en effet de placer cette colonne à proximité immédiate du cap Kainys qui formait la pointe de l'Italie, donc juste en face du cap Pélôros ou Pélôrias qui fermait le détroit du côté sicilien ${ }^{12}$. Cette situation au point le plus resserré du détroit, sans doute aux environs des villes actuelles de Cannitello ou Villa San Giovanni, fait de cette colonne un des sites possibles de la traversée vers la Sicile, le traiectus ad Siciliam mentionné à la fin du parcours de la poste publique d'époque impériale, le cursus publicus, en Calabre ${ }^{13}$. Strabon identifie en outre cette colonne à un ancien sanctuaire de Poséidon qui aurait marqué la limite septentrionale du territoire de Rhégion, à cent stades de la cité ${ }^{14}$. Si l'on retient cette information, la présence d'un sanctuaire de Poséidon en un lieu qui est à la fois l'entrée du détroit, son point le plus étroit et le lieu de sa traversée, ne peut manquer d'attirer l'attention, d'autant plus qu'il est couplé à un autre monument significatif du côté sicilien.

6 Le cap Pélôros ou Pélôrias tenait en effet son nom de la présence d'un monument ou tombe (mnêma) de Pélôros, parfois qualifié aussi de tour, pyrgos, situé sur le promontoire, juste en face de la colonne ${ }^{15}$. Une tradition faisait de ce Pélôros un pilote grec qui avait été chargé par les Carthaginois de conduire leur flotte entre la Sicile et le continent mais qui, accusé par eux de les avoir menés dans une impasse parce qu'ils avaient cru que le détroit était un golfe fermé, fut mis à mort; ayant cependant 
constaté leur erreur, les Carthaginois auraient enseveli le pilote bien en vue sur le cap ${ }^{16}$. Mais Francis Vian avait déjà reconnu le caractère évhémériste de ce récit en soulignant qu'en présence de Poséidon, il est difficile de ne pas rapprocher ce Pélôros, figure que son nom suffit à désigner comme "gigantesque ", du Pélôr ou Pélôros associé à l'une des entreprises les plus célèbres du dieu ébranleur de la terre : l'ouverture de la vallée du Tempé pour permettre aux eaux qui recouvraient la plaine de Thessalie de former le fleuve Pénée et de s'écouler vers la $\operatorname{mer}^{17}$. Ce séisme est souvent rapporté au culte thessalien de Poséidon Pétraios ou Lytaios, deux épiclèses pouvant faire allusion, la première à l'action fracassante du dieu sur les montagnes, la seconde à l'effet libérateur du séisme pour les eaux de Thessalie ${ }^{18}$. Mais il est également mentionné dans la notice qu'Athénée consacre à la célébration de la fête thessalienne des Pélôria, notice sans doute gauchie par l'usage que l'auteur des Deipnosophistes en fait pour montrer que les Grecs avaient des fêtes semblables aux Saturnales romaines, mais qui met néanmoins clairement en rapport Pélôros avec l'ouverture du Tempé ${ }^{19}$. C'est en effet « un homme appelé Pélôros » qui, lors d'une fête des Pélasges thessaliens, vient annoncer à leur roi Pélasgos qu'un tremblement de terre a créé un passage dans les montagnes et permis l'écoulement des eaux ; aussitôt le roi donne sa propre place à Pélôros et, avec tous les notables, se met à le servir - geste initial servant à expliquer l'inversion généralisée des positions sociales qui caractériserait la fête. On voit bien qu'ici aussi, une forme de banalisation de Pélôros, réduit à l'état de messager humain, est nécessaire pour en faire un personnage quelconque soudainement et provisoirement hissé au rang royal. Mais cette réduction ne peut cacher la relation du personnage avec Poséidon : c'est bien le séisme causé par le dieu que Pélôros vient annoncer, et un autre récit l'associe au fleuve Sperchéios où, en tant que géant, il tente d'assaillir la nymphe Polydôra, mais est tué par Poséidon qui le transperce de son trident ${ }^{20}$. L'ambiguïté est un trait récurrent des personnages monstrueux associés à Poséidon, comme on pourra le constater ultérieurement : beaucoup peuvent être présentés tantôt comme nocifs, tantôt comme bienfaisants. Ce trait ne saurait surprendre: il est déjà présent dans la Théogonie d'Hésiode où les Cent-Bras, d'abord adversaires terribles des Olympiens, finissent par se rallier à eux et aident plus particulièrement Poséidon à maintenir les Titans dans leur prison du Tartare - la proximité entre le dieu et les Cent-Bras se traduisant par l'idée que Briarée épousa une fille de Poséidon ${ }^{21}$.

7 La double présence de Pélôros auprès de Poséidon en Thessalie et sur le détroit de Messine prend tout son sens lorsqu'on met en parallèle les récits sur l'ouverture du Tempé et l'ouverture du détroit. Celle-ci est en effet pensée elle aussi comme le résultat d'un séisme, en partie en raison d'un rapprochement étymologique entre le nom de Rhégion et le verbe rhêgnumi, comme chez Diodore de Sicile où l'ouverture du détroit est présentée comme le résultat de l'assaut des vagues des deux côtés de l'isthme étroit qui raccordait la Sicile au continent et qui finit par être creusé ( $\dot{\alpha} v \alpha \rho \rho \alpha \gamma \tilde{\eta} v \alpha l)$. Mais Diodore, et avec lui Strabon (qui cite Eschyle) ajoutent que d'autres récits présentent la rupture de l'isthme comme la conséquence de grands tremblements de terre qui brisèrent $(\delta 1 \alpha \rho \rho \alpha \gamma \tilde{\eta} v \alpha)$ le "cou » entre l'île et le continent ${ }^{22}$. On se trouve ainsi en pleine entreprise poséidonienne de rupture visant à laisser passer les eaux, la cassure $\mathrm{du}$ détroit sicilien étant l' équivalent pour la circulation des eaux marines de la cassure des montagnes thessaliennes pour la circulation des eaux fluviales. Que Pêloros soit présent dans les deux cas, indépendamment des aspects variables sous 
lesquels il apparait, souligne cette analogie de situation. Mais il n'est pas le seul géant poséidonien à apparaître dans les récits rhégiens.

8 Diodore en effet rapporte une autre tradition encore, rapport ée à Hésiode, qui semble à première vue contradictoire avec les précédentes : le détroit aurait été créé non pas en cassant un isthme, mais au contraire en rapprochant les deux rives ${ }^{23}$. Le continent et la Sicile auraient été séparés par une grande étendue d'eau, et c'est pour les rapprocher que le géant Orion aurait édifié le promontoire Pélôris. L'opération est elle aussi sous le patronage de Poséidon : après avoir terminé son ouvrage, Orion « construisit le sanctuaire de Poséidon qui est tenu en très grand honneur par les habitants du lieu ». Il ne fait guère de doute qu'il s'agit du sanctuaire identifié à la "colonne de Rhégion ${ }^{24}$. L'intervention d'Orion s'inscrit dans un ensemble de travaux d'aménagement qu'il effectue dans la région du détroit pour le compte du roi Zanclos dont il s'est fait le serviteur: à Zancle même il crée le port enconstruisant un autre promontoire, l'Actê ${ }^{25}$. Mais l'action d'Orion a aussi pour effet de renforcer la présence de Poséidon dans les traditions du détroit. Orion est parfois considéré comme étantfils du dieu, qui lui aurait donné le pouvoir de franchir les mers en marchant ${ }^{26}$. Et le personnage a toute l'ambiguïté propre aux géants de Poséidon: si la légende devenue canonique, telle qu'elle est rapportée par exemple par Parthenios ou le Pseudo-Apollodore, a plutôt tendance à mettre en avant les manifestations d'hybris d'Orion, il y a bien d'autres traditions, notamment chez Corinne de Tanagra, où le géant, qu'il soit d'origine divine ou humaine, s'insère sans difficulté dans la lignée des rois, fondateurs et conquérants de Béotie orientale. Natif d'Hyria ou de Tanagra, Orion y apparaît souvent comme unhéros bienfaiteur, voire eusêbestatos, qui a mission, entre autres, d'exterminer les animaux sauvages pour rendre de nouvelles régions habitables ${ }^{27}$.

9 La double présence de Pélôros et Orion peut sembler contradictoire dans la mesure où l'un des géants est associé plutôt à la rupture des terres - en l'occurrence, l'isthme qui reliait les deux rives, ce qui éloigne celles-ci l'une de l'autre - , tandis que l'autre inversement les rapproche en édifiant un promontoire qui resserre le détroit. Cette contradiction apparente n'est pas spécifique au détroit de Sicile : elle caractérise aussi les récits relatifs à la création des colonnes d'Héraclès. Dans le récit que Diodore de Sicile consacre aux exploits du héros sur le détroit de Gibraltar, celui-ci se voit attribuer les deux mêmes types d'action: soit il construit deux promontoires qui réduisent la distance entre la Libye et l'Ibérie, facilitant ainsi le passage de l'une à l'autre et bloquant l'accès à la Méditerranée aux grands monstres océaniques; soit il sépare les deux continents qui étaient jusque-là reliés l'un à l'autre en ouvrant le passage entre les deux, ce qui a pour effet de mélanger l'eau de l'océan à l'eau de la mer intérieure ${ }^{28}$.

10 Le parallèle avec le détroit de Messine n'est évidemment pas fortuit ; le rapprochement entre les deux passages maritimes avec leurs colonnes respectives est d'ailleurs explicite chez Strabon ${ }^{29}$. Mais la combinaison des deux types d'action - briser l'obstacle terrestre pour ouvrir le passage aux flots marins, ou rapprocher les promontoires pour faciliter le passage d'une rive à l'autre -, loin d'être une contradiction, est constitutive de la double fonction des détroits. Ceux-ci doivent en effet être assez larges et sans obstacle pour laisser libre cours à la navigation longitudinale, la traversée le long du détroit, que je qualifierai ici, par pure convention, de poros; mais ils doivent aussi être assez resserrés pour permettre la traversée latitudinale, d'un bord à l'autre, que 
j'appellerai, de manière tout aussi conventionnelle, le porthmos ${ }^{30}$. La double action des dieux, géants et héros œuvrant sur les détroits vise donc à donner à ceux-ci leurs justes dimensions du point de vue des deux types de navigation qui s'y croisent et, de ce fait, loin d'être contradictoire, elle est en fait parfaitement complémentaire. Cela démontre bien qu'on ne peut penser les séismes poséidoniens sous la seule catégorie de la catastrophe, même s'ils en revêtent souvent la forme ${ }^{31}$. Il n'est pas inintéressant de noter que cette conception survivra de beaucoup à l'Antiquité : dans les sources arabes médiévales qui reportent d'Héraclès sur Alexandre la création du détroit de Gibraltar, et dont certaines suivent de très près le récit de Diodore, le conquérant se voit attribuer de la même manière une double action d'ouverture ou de fermeture, ainsi que l'érection d'une statue (visiblement inspirée d'une grande statue du sanctuaire d'Héraclès de l'antique Gadès, en place jusqu'au XIII ${ }^{e}$ siècle) qui remplace les « colonnes » et dont le bras tendu, selon les versions, indiquait ou interdisait l'accès au passage $^{32}$.

\section{Poséidon et le « tenir ensemble »}

11 Le rapprochement avec les colonnes d'Héraclès attire l'attention sur une autre singularité. Si, pour les auteurs arabes qui n'avaient plus qu'une idée assez vague d'Héraclès, la légende d'Alexandre offrait un héros de substitution tout à fait convaincant pour procéder à la " clôture du monde » en érigeant des statues de garde aux confins de l'oecoumène, il se peut aussi qu'Héraclès lui-même ait supplanté un autre créateur de détroits. Certaines traditions évoquent en effet la présence de Briarée sur le détroit de Gibraltar. Plusieurs fragments des poètes hellénistiques Euphorion et Parthenios, transmis par le biais de scholies, mentionnent des "colonnes de Briarée » (ou dans un cas d'Aigaion, mais on verra plus loin que cela ne change rien de fondamental) qui auraient précédé les colonnes d'Héraclès ${ }^{33}$. Selon un scholiaste à Denys le Périégète dont la source est Euphorion, les colonnes érigées sur le détroit auraient même été appelées successivement colonnes de Cronos, car elles marquaient les limites de son royaume, puis colonnes de Briarée, et enfin colonnes d'Héraclès ${ }^{34}$.

Cette tradition a été mise en relation avec une très ancienne présence eubéenne à l'entrée de la Méditerranée, dès le viII ${ }^{\mathrm{e}}$ siècle ${ }^{35}$. Assimilé au Cent-Bras de la Théogonie, fils de Terre et de Ciel dont une tradition fait aussi le gendre de Poséidon, Briarée est en effet un géant dont la légende est fortement enracinée en Eubée ${ }^{36}$. Il y apparaît sous le nom de Briarée, de Briarée-Aigaiôn, ou simplement Aigaiôn, cette diversité d'appellations trouvant une explication dans le passage de l'Iliade où Thétis envoie sur l'olympe « l'être aux cent bras que les dieux nomment Briarée et les hommes Aigaion, et qui par la force surpasse son père même $\aleph^{37}$. Par-delà la diversité des dénominations, les récits dessinent la figure d'une puissance du domaine maritime, soit en qualité de " dieu marin » (enalios theos chez Hésychius ou enalios daimôn dans une scholie à l'Iliade), soit comme souverain d'une thalassocratie centrée sur l'Eubée, tantôt à Chalcis, tantôt à Carystos, et s'étendant sur les Cyclades et la mer à laquelle il aurait donné son nom, l'Égée ${ }^{38}$. L'ancrage eubéen de ces récits est évidemment renforcé par le fait que sur le détroit de Sicile, encadré par les deux fondations eubéennes de Rhégion et Zancle, Orion et Pélôr appartiennent à des univers culturels eubéens ou proches de l'Eubée (thessalien et béotien). Dans le récit rapporté par Diodore qui fait d'Orion le constructeur du cap Pélôrias, le géant, son travail accompli, « retourna en Eubée et y 
établit sa demeure $»^{39}$. La légende d'Orion semble de ce fait établie sur les deux rives de l'Euripe. Peut-on, ou même doit-on, nécessairement rapporter toutes ces histoires de géants au milieu culturel eubéen? C'est peut-être se montrer trop restrictif, comme nous le verrons. En l'état actuel des choses, l'hypothèse d'une présence eubéenne sur le détroit de Gibraltar à haute époque est invérifiable; et le fait que les sources fréquemment citées de l'histoire de Briarée soient deux poètes d'époque hellénistique invite à une certaine prudence. Mais l'intérêt de la présence de Briarée et de ses colonnes ne dépend pas fondamentalement de la réalité ou non de la présence eubéenne; même en tant qu'invention poétique, elle est signifiante et éclaire la conception que l'on pouvait se faire du Cent-Bras. En particulier, la tradition selon laquelle les colonnes du détroit de Gibraltar avaient été nommées successivement colonnes de Cronos, colonnes de Briarée, puis colonnes d'Hercule installe le géant dans une position intermédiaire entre l'âge de Cronos et le règne de Zeus, dont nous verrons qu'elle correspond très exactement au rôle "théologique " de Poséidon et à ses interventions sur les détroits.

On ne s'écartera cependant pas de l'Eubée sans examiner au préalable les traditions concernant le détroit de l'Euripe. Outre le nom « humain » de Briarée, Aigaiôn est aussi l'épiclèse donnée à Poséidon dans son sanctuaire d'Aigai d'Eubée ${ }^{40}$. Pour se rattacher à l'épisode de l'Iliade où le dieu, descendant du sommet de la montagne de Samothrace, " fait trois enjambées et, à la quatrième atteint son but, Aigai, où un palais illustre lui a été construit dans les profondeurs de la mer ", deux cités, chacune avec son sanctuaire de Poséidon, se disputèrent l'honneur d'être l'Aigai homérique : Aigai d'Achaïe et Aigai d'Eubée, Strabon donnant la préférence à cette dernière ${ }^{41}$. Le géographe précise aussi que cette Aigai d'Eubée se trouve en face de la portion de la côte béotienne qui, d'Anthédon à Larymna, s'étend au nord du détroit de l'Euripe. Or, c'est entre Anthédon et l'Euripe que se trouvait, sur une hauteur, le tombeau de Salganeus, un monument dont l'histoire est identique à celle du tombeau de Pélôros en Sicile. Salganeus est en effet un Béotien qui aurait conduit la flotte perse depuis le golfe maliaque jusqu'au détroit de l'Euripe. Aussi aveugles que les Carthaginois, les Perses auraient cru que leur pilote les avait emmenés au fond d'une nasse et l'auraient mis à mort. Mais quand il se rendit compte de leur erreur, leur navarque Mégabatos «se repentit et jugea digne d'un tombeau celui qu'il avait mis à mort sans raison $\gg^{42}$. Le parallélisme entre les mésaventures des deux pilotes est évidemment trop parfait pour ne pas être construit. Il est caractéristique de la mise en miroir effectuée par l'historiographie grecque entre les agressions perses à l'est et carthaginoises à l'ouest. Mais dans la mesure où la légende de Pélôros résulte vraisemblablement d'une interprétation evhémériste d'un géant proche de Poséidon, associé à la mer et au creusement du détroit de Sicile, on peut supposer qu'il en va de même pour Salganeus et que ce dernier formait, avec le Poséidon Aigaiôn dont le sanctuaire se trouvait en face, sur la côte eubéenne, un duo tout à fait analogue à celui que formaient Pélôros et le sanctuaire de Poséidon de la «colonne de Rhégion ». Les deux détroits de l'Euripe et de Sicile offriraient ainsi la même construction légendaire et cultuelle, bien que nos sources n'aient pas transmis de récit de l'ouverture/fermeture de l'Euripe.

14 Tous ces exemples permettent d'identifier un des ressorts fondamentaux de l'action de Poséidon sur les détroits et passages. Il ne suffit pas, en effet, de reconnaître l'action de l'ébranleur du sol dans les séismes, ruptures et ouvertures; le cas des détroits a bien montré que l'action de fermeture, parfois œuvre des géants compagnons du dieu, en est le complément nécessaire. Il s'agit donc d'un processus dont la fin ultime est la 
stabilisation des passages, et Poséidon apparaît ici comme le dieu qui « tient ensemble » - le gaiochos, si on veut, mais dans un sens bien précis : tenir ensemble et à la juste distance les deux rives d'un détroit, les deux côtés d'une vallée, ou encore tenir ensemble la mer et les terres, l'eau et la terre. Il s'agit donc plus précisément de «tenir ensemble le dissemblable ». Or, il est possible d'élargir cette analyse à d'autres types de passage et de paysage. Les isthmes vont à leur tour nous apporter des éléments de compréhension de l'action de Poséidon.

\section{Le joug, du rivage à l'attelage}

Un isthme, dans notre vision courante, est vu comme un pont reliant deux ensembles terrestres. L'isthme écarte les eaux situées de chaque côté pour faciliter le passage longitudinal d'une terre à l'autre. Cette conception existe évidemment chez les anciens Grecs. L'isthme de Corinthe, par exemple, peut être appelé "pont», gephyra. Ainsi Pindare parle-t-il en ces termes du Poséidon de l'isthme de Corinthe ${ }^{43}$ :

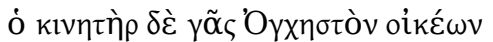

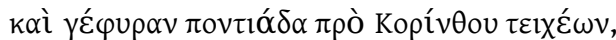

Le dieu qui ébranle la terre, qui réside à Onchestos et habite le pont jeté sur la mer aux abords des murs de Corinthe.

16 L'isthme de Corinthe est cependant connu pour avoir servi aussi de jonction entre le golfe saronique et le golfe de Corinthe, grâce aux deux ports que la cité avait établis de part et d'autre - autrement dit, pour un isthme comme pour un détroit, en reprenant la distinction établie plus haut de façon conventionnelle et en inversant le rapport entre terre et mer, il y a une fonction de porthmos, au sens de traversée latérale des terres, aussi bien que de poros, au sens de traversée longitudinale. Or cette homologie transparaît dans le vocabulaire utilisé pour parler de l'isthme, comme on peut le voir dans l'un des Tableaux de Philostrate consacré à l'histoire de Palaimon ${ }^{44}$ :

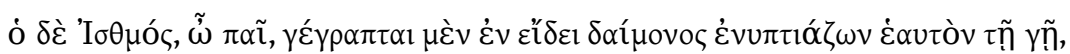

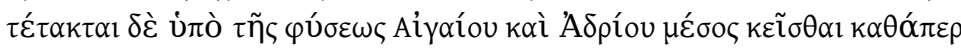

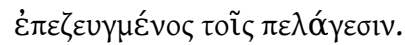

L'Isthme, enfant, est représenté sous la forme d'un dieu endormi, étendu par terre sur le dos. La nature lui a assigné de se tenir ainsi entre l'Égée et l'Adriatique, comme un joug placé sur les deux mers.

17 L'isthme, qui est de surcroît l'isthme où réside Poséidon, devient ici le joug qui tient ensemble les deux mers, ce qui amène immédiatement à l'esprit la comparaison avec un attelage à deux chevaux; autrement dit, autant qu'il sépare, l'isthme maintient, réunit les deux mers. Loin d'être un simple passage, l'isthme, autant que le détroit, relie et tient ensemble ce qu'il sépare. Cette fonctionnalité n'est donc pas réservée aux seuls passages maritimes : elle s'étend aux passages terrestres où, très clairement, Poséidon exerce cette fonction $\mathrm{du}$ « tenir ensemble » qui procède à la fois de la séparation et de la réunion, ou mieux encore d'une séparation qui est aussi réunion, deux aspects indissociables d'une même réalité qui garantit la stabilité des grands passages nécessaires aux voyages maritimes et terrestres.

Le passage de la IV Isthmique de Pindare cité précédemment permet d'aller un peu plus loin encore. Il associe en effet et met sur le même plan deux des lieux de résidence favoris de Poséidon: Onchestos en Béotie et l'Isthme de Corinthe ${ }^{45}$. Ce rapprochement appelle deux commentaires. Le premier concerne la configuration topographique d'Onchestos. Le sanctuaire est en effet associé à une chaîne de collines qui, se détachant 
du piedmont nord du massif de l'Hélicon, se dirige vers le nord-est, pratiquement jusqu'aux abords d'Acraiphia et du massif du Ptoion. Cette dorsale basse sépare ainsi les deux grands ensembles formant la Béotie : à l'est, la plaine de Thèbes; à l'ouest, la plaine d'Orchomène et le bassin du Copais. Le sanctuaire reste assez mal connu mais il est situé sur le parcours de la route qui vient de Thèbes et franchit ces collines par une passe, selon un tracé qui ne doit guère différer de celui de la route antique ${ }^{46}$. Situé quasiment à équidistance des deux grandes cités béotiennes, Onchestos est dans une position qui peut être à la fois centrale et frontalière. Récits et documents ne manquent pas qui soulignent le rôle de lieu de rencontre joué par le sanctuaire au niveau béotien, soit dans un cadre conflictuel comme celui des guerres entre Orchomène et Thèbes, soit au contraire dans le cadre de rassemblements pan-béotiens. Dans le premier cas, Onchestos est au cœur des traditions concernant le conflit entre les Minyens d'Orchomène et les Thébains : c'est lors des fêtes de Poséidon, auxquelles les deux peuples participaient, que le roi Clymenos d'Orchomène est blessé ou tué par un Thébain, ce qui déclenche la guerre ${ }^{47}$. Le second cas est illustré par le rôle qu'Onchestos joua comme capitale de la Confédération béotienne de la destruction de Thèbes en 335 à la dissolution du koinon en 172. Le Poséidon d'Onchestos possède donc lui aussi cette double fonction de séparation et d'assemblage, et ce trait invite à établir un parallèle entre la ligne de collines où il se trouve et l'isthme de Corinthe : de la même manière que ce dernier tout à la fois divise et maintient ensemble les deux mers qui l'encadrent, on peut dire que la dorsale où se trouve le sanctuaire d'Onchestos sépare et tient ensemble les deux plaines qui la bordent. Et c'est là que le second aspect du rapprochement entre les deux paysages prend tout son sens. L'isthme, avons-nous vu, "met sous le joug" les deux mers, les met en attelage. Or, où sinon à Onchestos la métaphore de l'attelage prend-elle tout son sens? Toutes les traditions relatives au sanctuaire parlent d'attelages et de courses de chars. Dans les récits des origines des guerres minyennes, c'est souvent lors de courses de chars que Clyménos est tué. Mais le plus important est évidemment le célèbre passage de l'Hymne homérique à Apollon décrivant un rite tout à fait spectaculaire ${ }^{48}$ :

De là poussant plus avant, Archer Apollon, tu as atteint Onchestos, bois resplendissant de Poséidon. C'est là que le poulain nouvellement dompté reprend son souffle, bien qu'il reste chargé du poids du char, et si habile qu'il soit, le conducteur saute à terre et continue la route à pied. Une fois qu'ils ne sont plus tenus en main, les chevaux font résonner le char vide. Si le char se brise dans le bois touffu, les conducteurs soignent les chevaux et laissent le char après l'avoir incliné.

C'est ce qui fut autorisé dès l'origine par la loi sainte ; on invoque le seigneur et c'est le lot du dieu de protéger le char.

Ce texte et le rite qu'il évoque ont évidemment suscité de nombreux commentaires mais, pour les besoins de la démonstration, je retiens surtout celui qu'en avaient donné Marcel Detienne et Jean-Pierre Vernant dans leur ouvrage sur la mêtis ${ }^{49}$. Attachés qu'ils étaient à montrer que dans les domaines requérant un savoir-faire précis, comme la construction et la conduite des navires ou le domptage des chevaux et la conduite des chars, la véritable maîtresse de la technê, la seule détentrice de la mêtis était Athéna, les deux auteurs n'ont laissé à Poséidon qu'un rôle limité, assez passif, relevant bien plus de l'exercice de la force et de la puissance que de la mise en œuvre de l'habileté ${ }^{50}$. Pour le rite d'Onchestos, ce rôle était même défini comme "essentiellement négatif " puisque le dieu se limiterait à décider s'il effraye ou non le cheval lancé dans sa course. Mais il est clair qu'on ne peut plus s'en tenir à cette analyse. De manière générale, la relation entre Athéna et Poséidon est beaucoup plus riche, variée et nuancée que ce qui 
est présenté dans l'ouvrage, où le second est réduit au rôle de simple faire-valoir de la première. Au moins sur ce point et sur les fonctions de Poséidon, les analyses de Detienne et Vernant doivent être partiellement revues, en cherchant à mettre en valeur ce qui rend Athéna et Poséidon complémentaires plutôt qu'opposés ${ }^{51}$. En particulier, on ne peut maintenir que ce qui relève du joug, de l'attelage et de l'art de la charrerie relève essentiellement d'Athéna. C'est oublier que Poséidon peut être qualifié de "dieu du joug", zugios; et c'est ne pas tenir compte de notices comme celle qu'Hésychius rapporte, d'après Mnaseas de Patras, quand il écrit que les Libyens « ont été les premiers à apprendre de Poséidon l'art d'atteler des chars, et d'Athéna l'art de conduire les chars $\aleph^{52}$. Cette répartition des compétences permet d'éclairer d'un autre jour le rite accompli dans le sanctuaire d'Onchestos. Elle indique en effet que, dès que le cocher descend du char, ce dernier sort du domaine de la conduite de l'attelage, et par conséquent ne se trouve plus dans le champ d'action d'Athéna. Resté seul avec le char, le cheval (ou les chevaux s'il s'agit d'un attelage) ne relève plus que de l'art d'atteler les chars; il entre donc pleinement dans le domaine de Poséidon. L'épreuve rituelle vise donc à tester non pas, ou pas uniquement, le comportement du cheval, pour savoir s'il a été bien dressé ou non, mais aussi, voire avant tout, la solidité et la qualité de l'attelage formé par le cheval ou l'attelage et le char vide. Autrement dit, c'est la qualité de l'assemblage, du joug qui est mis à l'épreuve. C'est en cela que le rite convient parfaitement à Poséidon, qui préside ainsi de nouveau à l'efficacité du «tenir ensemble ", et on comprend parfaitement que les chars des attelages qui n'ont pas su faire la preuve de leur solidité restent dans le sanctuaire, consacrés au dieu.

On voit ainsi se mettre en place une articulation parfaitement cohérente entre le rite d'Onchestos et le rôle du sanctuaire comme «joug » des deux parties de la Béotie, au même titre que l'isthme de Corinthe est le joug des deux mers. Et par delà ces deux exemples, c'est toute la série des cas analysés ici qui trouve sens en faisant du dieu l'agent et le garant d'un "tenir ensemble» s'exerçant sur un vaste ensemble de passages, d'isthmes et de détroits, tous paysages qui, combinant séparation et réunion, ne peuvent exister et prendre sens que s'ils sont tenus « sous le joug " par Poséidon. On voit bien que l'étendue de cette compétence de Poséidon va bien au-delà du sens traditionnellement assigné à l'épithète gaiochos. Il ne s'agit pas seulement de "tenir ", mais de tenir réunis des éléments qui sont souvent dissemblables : caps et passages maritimes dans les détroits, mers et passage terrestre sur les isthmes, eaux et montagnes dans les vallées, chevaux et chars, régions à la fois solidaires et rivales de part et d'autre d'une séparation topographique... Comment expliquer ce rôle si particulier de Poséidon qui acquiert ainsi, dans l'aménagement du monde, un rôle essentiel et bien plus dynamique que celui qui lui est souvent accordé ?

\section{Poséidon, seigneur des deux mondes}

21 J'ai déjà relevé que la tradition rapportée à Euphorion selon laquelle les colonnes érigées sur le détroit à l'entrée de la Méditerranée se seraient appelées successivement colonnes de Cronos, colonnes de Briarée et colonnes d'Héraclès installe le Cent-Bras à la jonction entre l'âge de Cronos et le règne de Zeus. Or cette position intermédiaire correspond bien à la position du géant, et avec lui, au rôle que joue Poséidon dans la Théogonie ${ }^{53}$. D'abord adversaires des Olympiens, les Cent-Bras passent ensuite à leur service et les aident à vaincre les Titans en écrasant ces derniers sous des masses de 
rochers; et lorsque les Titans sont enfermés dans le Tartare derrière une enceinte de bronze, c'est Poséidon qui referme sur eux les portes de métal où les Cent-Bras vont continuer à monter la garde. En tant que fils d'Ouranos et de Gaia, les Cent-Bras appartiennent pleinement à l'âge de Cronos; mais en même temps ils contribuent activement à l'instauration du règne de Zeus et y gardent une fonction de maintien des fondements du monde en surveillant le Tartare. Et c'est là qu'ils sont au plus près de Poséidon qui bâtit les portes du Tartare et fait de Briarée son gendre. L'association du dieu et des géants trouve ainsi ses origines dans un récit qui installe leur complicité au moment crucial où s'effectue le passage entre deux règnes. D'une certaine façon, les Cent-Bras et Poséidon avec eux sont à cheval sur deux mondes, deux âges qu'ils tiennent ensemble pour effectuer la transition et assurer la stabilité du règne de Zeus. Cette dualité peut expliquer le double visage des géants associés à Poséidon, tantôt redoutables, tantôt serviables, comme s'ils portaient les caractères distinctifs des deux âges auxquels ils appartiennent. Mais de façon générale, elle donne un fondement "théologique " au "tenir ensemble» que Poséidon exerce dans de nombreuses situations, un tenir ensemble qui s'exerce sur des éléments souvent dissemblables et qui, loin de confiner le dieu dans l'exercice de la seule force, dans la manifestation brutale d'une colère destructrice, ou dans la survivance d'une souveraineté disparue, fait du dieu l'artisan de bien des équilibres, non seulement dans le domaine tellurique, mais aussi dans les savoir-faire où il rencontre Athéna, et jusque dans le domaine politique.

\section{BIBLIOGRAPHIE}

L. BREGLIA PULCI DORIA, « La Sardegna arcaica tra tradizioni euboiche ed attiche », in Nouvelle contribution à l'étude de la société et de la colonisation eubéennes, Cahiers du Centre Jean BérardVI, Naples, 1981, p. 61-92.

N. CASTRIZIO, « Note di iconografia siciliota (II). La statua dello stretto », Polifemo. Rassegna bibiografica di Storia delle religioni e Storia antica VII (2007), p. 211-222.

S. CROGIEZ, «Les stations du cursus publicus en Calabre : un état de la recherche », MEFRA 102 (1990), p. 389-431.

M. DETIENNE, Apollon le couteau à la main, Paris, 1998.

M. DETIENNE, J.-P. VERNANT, Les ruses de l'intelligence. La mètis des Grecs, Paris, 1974.

C. DOYEN, Poséidon souverain. Contribution à l'étude religieuse de la Grèce mycénienne et archaïque, Bruxelles, 2011.

M. GRAS, La Méditerranée archaïque, Paris, 1995.

P. ELLINGER, Artémis, déesse de tous les dangers, Paris, 2009.

D. JAILlARD, Configurations d'Hermès. Une théogonie hermaïque, Liège, 2007 (Kernos, suppl. 17).

J.-M. KoWALSKI, Navigation et géographie dans l'Antiquité gréco-romaine, Paris, 2012. 
L. LERAT, « Géraistos et les Géraistai », RA (1946), p. 196-203.

A. MELE, « Discussion », in Nouvelle contribution à l'

étude de la société et de la colonisation eubéennes, Cahiers du Centre Jean BérardVI, Naples, 1981, p. 138139.

L. MERCURI, « Les Eubéens et la colonne de Rhégion », MEFRA 110 (1998), p. 551-569.

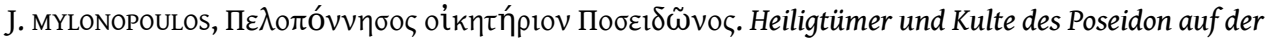
Peloponnes, Liège, 2003 (Kernos, suppl. 13).

V. PIRENNE-DELFORGE, G. PIRONTI, L’Héra de Zeus. Ennemie intime, épouse définitive, Paris, 2016.

G. PIRONTI, Entre ciel et guerre. Figures d'Aphrodite en Grèce ancienne, Liège, 2007 (Kernos, suppl. 18).

F. DE POLIGNAC, « La construction de l'universalité dans la légende d'Alexandre », Mètis n.s. 1

(2003), p. 251-269.

F. DE POLIGNAC, in Annuaire de l'EPHE, Section des Sciences religieuses 124 (2015-2016) [2017], p. 111113.

F. DE POLIGNAC, J. SCHEID (éd.), Qu'est-ce qu'un « paysage religieux » ?, dossier de la RHR 227 (2010), p. 427-719.

P. ROESCH, Études béotiennes, Paris, 1982.

F. SCHACHERMEYR, Poseidon und die Entstehung des griechischen Götterglaubens, Berne, 1950.

A. SCHACHTER, cults of Boiotia. 2. Heracles to Poseidon, Londres, 1986.

F. VIAN, « Géants de la mer », RA (1944), p. 97-117.

F. ViAN, « Génies des passes et des défilés », RA (1952), p. 129-155.

\section{NOTES}

1. VIAN (1944); VIAN (1952).

2. VIAN (1944), p. 116.

3. LERAT (1946).

4. VIAN (1952), p. 155.

5. Sur la notion de « paysage religieux », voir DE POLIGNAC, SCHEID (2010).

6. DETIENNE (1998) ; JAILLARD (2007) ; PIRONTI (2007) ; ELLINGER (2009) ; PIRENNE-DELFORGE - PIRONTI (2016).

7. SCHACHERMEYR (1950).

8. DOYEN (2011), dont les deux formules suivantes résument bien la pensée : « Poséidon mycénien, souverain en exercice », p. 257, et « Poséidon archaïque, souverain en relégation », p. 287.

9. DETIENNE - VERNANT (1974). Voir infra.

10. MYlonopoulos (2003), p. 373-440.

11. Strabon, III, 5, 5 (C 170), d'après Posidonios ; VI, 1, 5 (C 257) ; 2, 1 (C 265) ; Pomponius Méla, II, 68 ; Pline, NH III, 71, 73 et 86. Le dossier de la «colonne de Rhégion » a été étudié à fond par MERCURI (1998).

12. Strabon, VI, 1, 5 (C 257) donne une longueur de six stades à la portion de côte entre la colonne et le cap Kainys.

13. CROGIEZ (1990), p. 429-430.

14. Strabon, VI, 1, 5 (C 257). CASTRIzIo (2007) restitue une statue colossale de Poséidon au sommet de cette colonne, malgré le silence des sources classiques à ce sujet. 
15. Mnêma: Strabon I, 1, 16 (C 10) ;Pyrgos : Strabon III, 5, 5 (C 170).KowALSKI (2012), p. 104-111, consacre quelques pages au rôle des tombeaux et des tours comme « amers artificiels ».

16. Strabon, I, 1, 16 (C 10) ; Pomponius Méla, II, 7 ; Valère Maxime, IX, 8, parle d'une statue de Pélôros placée au sommet d'un haut tumulus.

17. Hérodote, VII, 129. VIAN (1952), p. 140-141.

18. Poséidon Pétraios : scholie Pindare, Pyth. IV, 246 (Drachmann); scholie Apollonios de Rhodes,

III, 1244 (Lachenaud) ; Poséidon Lytaios : Bacchylide, 18 (Thésée), 138 ; Étienne Byz., s.v. Lytai.

19. Athénée, XIV, 639d-640a.

20. Scholie Iliade XVI, 176.

21. Hésiode, Théogonie, 729-735; 807-819.

22. Diodore Sic., IV, 85, 3-5 ; Strabon, VI, 1, 6 (C 258).

23. Diodore Sic., IV, 85, 5 (= Hésiode, fr. 149 M.-W.).

24. Il est en effet peu vraisemblable, comme l'avance MERCURI (1998), p.564, qu'il y ait eu un second sanctuaire du dieu, sur le cap Pélore; la phrase de Diodore est trop vague pour en conclure à une localisation du sanctuaire créé par Orion sur le cap qu'il venait de mettre en place. 25. Diodore Sic., IV, 85, 1.

26. Parthénios, Souffrances de l'amour, 20, «Leirô ", 1-2 (Lightfoot) ; pseudo-Apollodore, I, 4, 2 (d'après Phérécyde) ; scholies Iliade II, 496.

27. Corinne (Campbell, Loeb 1991, IV), Les filles d'Asopos, fr. 654, v. 35-39 (Orion de retour sur ses terres reçoit, après Euonymos et Hyriée, l'honneur de rendre des oracles sur les trépieds d'Apollon) ; fr. 655, v. 14 (Orion et ses 50 fils) ; Le retour, fr. 662 (Orion conquérant) et fr. 673 (d'après la scholie à Nicandre, Theriaca, 15 : Orion né à Tanagra, "très pieux » et grand chasseur d'animaux sauvages). Orion natif d'Hyria de Béotie : Strabon, IX, 2, 12 ; scholia vetera Iliade XVIII, 486, où Orion, fils d'Hyriée, naît en fait de la semence mêlée de Poséidon, Zeus et Hermès.

28. Diodore Sic., IV, 18, 4-5. Voir aussi scholie Pindare, Ném. III, 38 (Drachmann).

29. Strabon, III, 5, 5 (C 170).

30. L'emploi des deux termes est ici conventionnel pour faciliter l'analyse. Poros et porthmos peuvent bien évidemment s'appliquer à un éventail beaucoup plus large de navigations, de passages et de traversées: voir KOWALSKI (2012), p.197-199. Sur les problèmes posés par les obstacles dans les détroits, on peut rappeler l'épisode bien connu de la colonne érigée par les navires éclaireurs perses pour signaler à la flotte de Xerxès les rochers à fleur d'eau qui se trouvaient au milieu du chenal entre la côte de la Magnésie et l'île de Skiathos (Hérodote, VII, 183).

31. Voir par exemple MYLONOPOULOS (2003), p. 391-395.

32. Ainsi chez Idrîsî et Ibn Khallikan (XIII ${ }^{\mathrm{e}}$ siècle), Maqrîzî (Xv ${ }^{\mathrm{e}}$ siècle) et al-Maqqarî (XVI ${ }^{\mathrm{e}}$ siècle) : voir DE POLIGNAC (2003), p. 251-253. Une légende analogue s'est développée à propos d'une statue qui, sur le détroit de Messine, aurait interdit la traversée vers la Sicile à Alaric en 410 : Olympiodore de Thèbes, in Photius, Bibliothèque, 80,57c. Ce récit renforce le parallélisme entre les deux détroits mais l'identification de cette statue magique à celle de Poséidon, comme le veut CASTRIZIO (2007), reste hypothétique.

33. Parthenius, fr. 34 (Lightfoot, Loeb 2009 = schol. Denys Perieg., 456). Voir aussi schol. Pindare, Ném. III, 40 (Drachmann) : les piliers d'Aigaion le géant, maître de la mer; et Hésychius, s.v. $\beta \rho \imath \alpha ́ \alpha \varepsilon \omega \sigma \tau \tilde{\eta} \lambda \alpha_{1}$ : stèles de Briarée, qui sont appelées d'Héraclès.

34. Euphorion, fr. 169 (Lightfoot = schol. Denys Perieg., 64).

35. BREGLIA PULCI DORIA (1981), p. 92 ; MELE (1981), p. 138-139; GRAS (1995), p. 16.

36. VIAN (1944), p. 168-169; MERCURI (1998), p. 565-566.

37. Iliade I, 402-404 (trad. Mazon, CUF). L'explication est reprise par Eustathe ad Iliad. I, p. 189-190

(v. 401-404). 
38. Hesychius, s.v. Aijoí $\omega v$; scholie Hom. Iliade I, 404a; Eustathe ad Iliad. I, p. 190 (v. 404) (thalassocratie). Solin, Collectanea Rerum Mirabilium, 11, 16 (Mommsen), place un culte de Briarée à Carystos et un culte d'Aigaiôn à Chalcis. Solin se fait l'écho d'une tradition qui fait de Briarée-

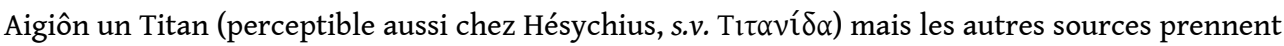
parfois la peine de réfuter cette identification (ainsi la scholie à Hom., Iliade I, 404a et Eustathe ad Iliad. I, p. 187 [v. 397]).

39. Diodore Sic., IV, 85, 5 .

40. Strabon, IX, 2, 13 (C 404-405).

41. Iliade XIII, 20-21 (trad. Mazon, CUF) ; Strabon, VIII, 7, 4 (C 385).

42. Strabon I, 1, 17 (C 10) ; IX, 2, 9 (C 403)

43. Pindare, Isthm. III/IV, 37-38 (trad. Puech, CUF).

44. Philostrate, Tableaux, 1, 16, 4 (Palaimon) (trad. pers. d'après la trad. Abbondanza, Milan 2008).

45. Pindare procède aussi à ce rapprochement dans Isthm. I, 32-33.

46. Deux ensembles ont été reconnus à un kilomètre de distance, l'un à l'est d'origine archaïque, l'autre plus à l'ouest en usage de la fin du IV siècle à l'époque romaine. ROESCH (1982), p. 268-276 ; SCHACHTER (1986), p. 207-219. Les fouilles ont repris très récemment sous la direction de Ioannis Mylonopoulos.

47. Pausanias, IX, 37 ; Ps.-Apollodore, II, 4, 11 ; Pap. Oxyr. 26. 2442, fr. 29.

48. Hymne homérique à Apollon, 229-238 (trad. Detienne et Vernant).

49. DETIENNE - VERNANT (1974), p. 193-195.

50. DETIENNE - VERNANT (1974), en particulier les deux chapitres « Le mors éveillé » et « La corneille de mer », p. 178-202 et 203-243.

51. C'est à cette révision qu'a été consacrée la première partie de ma conférence de l'EPHE en 2015-2016. Voir, provisoirement, DE POLIGNAC (2017).

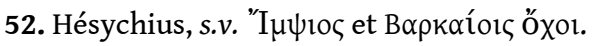

53. Hésiode, Théogonie, 711-735; 807-819.

\section{RÉSUMÉS}

Poséidon a été maintenu à l'écart de la plupart des travaux qui, s'inspirant de l'anthropologie historique, ont cherché à renouveler la conception des dieux grecs en montrant comment leur action dans le monde doit être comprise comme l'exercice d'une puissance dans des contextes dont les configurations dessinent des champs d'action multiformes. Le dieu reste souvent perçu comme une puissance déchue ou négative. La présente étude vise au contraire à mettre en lumière certains des ressorts, modalités et contextes des interventions de Poséidon en dessinant, par delà la diversité des situations, les contours d'un "paysage poséidonien » où apparaissent quelques traits fondamentaux des modes d'action du dieu. Passages, ouvertures et fermetures, maritimes et terrestres, révèlent les formes d'un «tenir ensemble » que le dieu, parfois en compagnie de géants, exerce en tenant « sous le joug » aussi bien les rivages que les attelages.

The study of Poseidon has not been much influenced by the scholarly works which, in the wake of the historical anthropology, deeply modified our views on the Greek gods by showing how their action in the world must be understood as a network of interrelated powers in varying contexts and multiform interventions. The god often remains perceived as a decayed sovereign or a negative power. By contrast, the present study aims at enlightening some of the motives, 
modes and contexts of Poseidon's activity through the identification, beyond each singular situation, of a "Poseidonian landscape » where some of the fundamental ways of acting of the god appear. Passages, openings and closings, either on sea or on land, thus reveal a capacity to « hold together » which the god, sometimes with the help of giants, exercises by « putting under the yoke » seas as well as horses.

\section{AUTEUR}

FRANÇOIS DE POLIGNAC

EPHE, PSL, UMR 8210 ANHIMA

francois.de-polignac@ehess.fr 